\title{
Comunidades de parásitos eumetazoos de peces labrisómidos de Chile central
}

\author{
Eumetazoan parasite communities of labrisomid fish from central Chile
}

\author{
Gabriela Muñoz ${ }^{1}$ y Raúl Castro²
}

\author{
${ }^{1}$ Facultad de Ciencias del Mar y de Recursos Naturales, Universidad de Valparaíso, Casilla 5080, Reñaca, Viña del Mar, Chile. \\ gabriela.munoz@cienciasdelmar.cl \\ ${ }^{2}$ Facultad de Recursos del Mar, Universidad de Antofagasta, Casilla 170, Antofagasta, Chile
}

\begin{abstract}
This study compares the parasite communities of 4 phylogenetically related fish species that belong to the family Labrisomidae; Auchenionchus microcirrhis, A. variolosus, A. crinitus and Calliclinus geniguttatus. These fish are sympatric in the intertidal zone of central Chile $\left(33^{\circ} \mathrm{S}\right)$, from where 182 specimens were collected. Twenty-one parasite taxa were found within the 4 sampled fish species. Three parasite species were the most common in the labrisomid fish: an undetermined leech (Piscicolidae), the copepod Holobomolochus chilensis, and the digenean Helicometrina nimia. Most of the parasites were host generalists. C. geniguttatus had a low similarity of parasite composition respect to Auchenionchus spp. The variation in parasite communities among labrisomid species were related to fish length and possibly to environmental variables.
\end{abstract}

Key words: Parasite infracommunities, Labrisomidae, congeneric fish species

\section{INTRODUCCIÓN}

Las comunidades de parásitos pueden ser caracterizadas por su composición de especies, basándose en la identidad taxonómica de cada integrante, así como en términos numéricos (abundancia, riqueza y diversidad). Estas características comunitarias pueden ser modificadas por numerosas variables relacionadas con aspectos ecológicos de los hospederos, como por ejemplo el tipo de hábitat, las condiciones ambientales, la dieta o su distribución, las que repercuten de alguna forma en la transmisión de los parásitos (e.g., Sapp \& Esch 1994, Thieltges et al. 2008).

Por otro lado, las características de las comunidades de parásitos tambien son influidas por la filogenia de los hospederos, ya que suelen heredar los parásitos de la especie de hospedero de la cual descienden, por lo tanto, especies emparentadas normalmente comparten parásitos específicos (Poulin 2007). Esto ha sido evidenciado en hospederos congenéricos ya que generalmente tienen similar composición parasitaria, aunque esas especies parásitas pueden variar en abundancia o prevalencia (e.g., Muñoz et al. 2006, McKenzie et al. 2011, Alarcos \& Timi 2012). Además, la composición de las comunidades de parásitos se modifica con el tiempo, es decir se pierden o se ganan especies parásitas debido a que los hospederos congenéricos paulatinamente cambian algún atributo ecológico, lo que repercute en la transmisión de parásitos
(Poulin 2007). Especialmente, especies de hospederos que han modificado algún aspecto biológico muy notorio, e.g., que se distribuyen en hábitats alejados (Díaz \& Muñoz 2010, Chávez et al. 2012), tienen una baja sobreposición de ítems alimentarios (Muñoz et al. 2006), o muestran un tamaño corporal y longevidad distinta (Alarcos \& Timi 2012) a la de sus congéneres, usualmente presentan una fauna parasitaria distinta.

Las especies de peces de la familia Labrisomidae son relativamente comunes tanto en la zona intermareal como en el submareal del litoral chileno y cuentan con varios antecedentes biológicos como distribución y dieta. Existen 4 especies en la zona central: Auchenionchus microcirrhis (Valenciennes, 1836), A. variolosus (Valenciennes, 1836), A. crinitus (Jenyns, 1842) y Calliclinus geniguttatus (Valenciennes, 1836). La distribución geográfica de las especies abarca gran parte de Chile; A. variolosus y C. geniguttatus se encuentran desde el norte al sur austral de Chile, mientras que $A$. microcirrhis y A. crinitus se distribuyen desde el sur del Perú hasta Chile central. Los labrisómidos son peces carnívoros que se alimentan principalmente de crustáceos decápodos, anfípodos e isópodos. La dieta de 3 especies analizadas hasta ahora (A. microcirrhis, A. variolosus y C. geniguttatus) han presentado más de un $65 \%$ de similitud cuantitativa (Muñoz \& Ojeda 1997, Quijada \& 
Cáceres 2000). La longitud corporal de labrisómidos de pozas intermareales oscila en un promedio de 6,4 y 9,6 cm (Muñoz \& Ojeda 1998, Quijada \& Cáceres 2000), siendo relativamente similares entre las especies. Estas similitudes biológicas (longitud corporal) y ecológicas (dieta y hábitat), sumándoles el grado de parentesco de estas 4 especies de labrisómidos, se esperaría que las comunidades de parásitos fuesen similares en composición y carga parasitaria entre las especies, especialmente entre los peces congenéricos.

A pesar del aumento progresivo de estudios parasitológicos en peces intermareales en la última década (e.g., Muñoz et al. 2002, Pardo-Gandarillas et al. 2004, Flores \& George-Nascimento 2009, Muñoz \& Cortés 2009, Moraga \& Muñoz 2010, Díaz \& Muñoz 2010, Muñoz \& Delorme 2011, Muñoz \& Randhawa 2011, Muñoz \& Zamora 2011), son escasos los estudios comunitarios en parásitos de peces labrisómidos y ausentes para las costas de Chile. Hasta el presente, solo existen estudios parasitológicos en Labrisomus philippii (Steindachner,
1866) realizados en las costas peruanas (Oliva \& Luque 2002, Iannacone et al. 2011). Por lo tanto, los objetivos del presente estudio fueron describir y comparar las comunidades de parásitos de 4 especies de labrisómidos de Chile central para determinar si las similitudes o diferencias se asocian a su grado de parentesco.

\section{MATERIALES Y MÉTODOS}

Los peces fueron recolectados desde pozas intermareales rocosas de Isla El Tabo (33⒉ $\left.7^{\prime} \mathrm{S}, 7^{\circ} 38^{\prime} \mathrm{O}\right)$ y Las Cruces

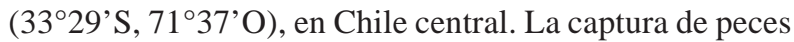
se realizó durante baja marea con la aplicación de anestésico BZ-20® y redes de mano, entre agosto de 2006 y julio de 2009. Durante ese tiempo se obtuvieron 182 ejemplares que correspondieron a 4 especies según las características morfológicas indicadas por Stephens \& Springer (1973); el 77\% de la muestra fue conformada por la especie Auchenionchus microcirrhis (Tabla 1).

Tabla 1. Prevalencia ( $P \%$ ) e intensidad promedio (y su desviación estándar, DE) de las especies parásitas encontradas en 4 especies de peces labrisómidos de Chile central / Prevalence (\%) and mean intensity (and standard deviation, DE) of the parasite species found in 4 labrisomid fish species of central Chile

\begin{tabular}{|c|c|c|c|c|c|c|c|c|c|c|c|c|}
\hline \multirow{2}{*}{ Taxa parasitarios } & \multicolumn{3}{|c|}{$\begin{array}{l}\text { Auchenionchus microcirrhis } \\
\qquad \mathrm{n}=141\end{array}$} & \multicolumn{3}{|c|}{$\begin{array}{l}\text { Auchenionchus crinitus } \\
\qquad \mathrm{n}=26\end{array}$} & \multicolumn{3}{|c|}{$\begin{array}{l}\text { Auchenionchus variolosus } \\
\qquad \mathrm{n}=8\end{array}$} & \multicolumn{3}{|c|}{$\begin{array}{l}\text { Calliclinus geniguttatus } \\
\qquad \mathrm{n}=7\end{array}$} \\
\hline & $\mathbf{P}$ & INT & $\mathrm{DE}$ & $\mathbf{P}$ & INT & $\mathrm{DE}$ & $\mathrm{P}$ & INT & $\mathrm{DE}$ & $\mathrm{P}$ & INT & $\mathrm{DE}$ \\
\hline \multicolumn{13}{|l|}{ HIRUDINEA } \\
\hline \multirow{2}{*}{\multicolumn{13}{|c|}{ COPEPODA }} \\
\hline & & & & & & & & & & & & \\
\hline Acanthochondria ophiidi & 2,1 & 9,0 & 13,0 & 3,8 & 1,0 & -- & 12,5 & 1,0 & -- & & & \\
\hline Lepeophtheirus zbigniewi & 12,1 & 4,8 & 6,0 & & & & 37,5 & 3,7 & 3,1 & 14,3 & 2,0 & .- \\
\hline Colobomatus tenuis & 6,4 & 1,9 & 1,3 & & & & & & & & & \\
\hline Holobomolochus chilensis & 41,1 & 3,1 & 3,1 & 30,8 & 1,6 & 1,1 & 62,5 & 3,4 & 2,8 & 14,3 & 1,0 & -- \\
\hline Trifur c.f. tortuosus & 2,1 & 1 & $0-$ & 7,7 & 1,0 & 0 & 37,5 & 1,3 & 0,6 & & & \\
\hline TURBELLARIA & & & & 7,7 & 1,0 & 0 & & & & & & \\
\hline \multicolumn{13}{|l|}{ MONOGENEA } \\
\hline Neobenedenia melleni & 0,7 & 1 & -- & & & & & & & & & \\
\hline Microcotyle sp. & & & & 3,8 & 2,0 & .- & 12,5 & 2,0 & -. & & & \\
\hline \multicolumn{13}{|l|}{ DIGENEA } \\
\hline Helicometrina nimia & 53,2 & 10,8 & 16,0 & 61,5 & 5,8 & 5,7 & 62,5 & 12,8 & 10,6 & 14,3 & 3,0 & -- \\
\hline Lecithaster $\mathrm{sp}$. & 7,1 & 1,3 & 0,5 & 7,7 & 1,0 & 0 & 12,5 & 1,0 & -- & & & \\
\hline Prosorhynchoides carvajali & 17,0 & 9,1 & 11,4 & & & & 25 & 70,0 & 94,7 & & & \\
\hline Hemipera sp. & 1,4 & 2,0 & 1,4 & & & & & & & & & \\
\hline Hemiuridae gen. sp. & & & & 3,8 & 1,0 & .- & 12,5 & 1,0 & 0 & & & \\
\hline \multicolumn{13}{|l|}{ CESTODA } \\
\hline Tetraphyllidea gen. sp. & 12,8 & 1,8 & 1,2 & 7,7 & 5,0 & 2,8 & 12,5 & 4,0 & -- & & & \\
\hline Pseudophyllidea gen. sp. & 1,4 & 5,3 & 4,0 & 7,7 & 3,5 & 2,1 & & & & & & \\
\hline Nybelinia sp. & 0,7 & 6,0 & -- & & & & & & & & & \\
\hline \multicolumn{13}{|l|}{ NEMATODA } \\
\hline Similascarophis chilensis & 0,7 & 4,0 & -- & & & & & & & & & \\
\hline Spirurida gen. sp. & 0,7 & 1 & -- & & & & 12,5 & 1,0 & -- & & & \\
\hline Anisakis sp. & 0,7 & 1 & - & & & & & & & & & \\
\hline \multicolumn{13}{|l|}{ ACANTHOCEPHALA } \\
\hline Corynosoma sp. & 13,5 & 1,5 & 0,6 & 26,9 & 1,9 & 1,5 & 25 & 1,0 & 0 & & & \\
\hline
\end{tabular}


Una vez que los peces fueron capturados, cada ejemplar fue depositado en una bolsa plástica. El 60\% de los ejemplares fueron luego congelados a $-10^{\circ} \mathrm{C}$ para su posterior análisis, mientras que el resto fue diseccionado en fresco con el propósito de obtener parásitos (sólo eumetazoos) en buenas condiciones para los procesos de tinción y de identificación. Cada pez fue medido en longitud total (según su abreviación LT) y pesado (peso húmedo total: PT). Luego, cada espécimen fue analizado externamente para la recolección de ectoparásitos y diseccionado para recolección de los endoparásitos con la ayuda de un microscopio estereoscópico. Los parásitos obtenidos fueron fijados y almacenados en formalina al $10 \%$ para su posterior determinación taxonómica. La identificación de parásitos fue realizada en base a preparaciones in toto teñidas con hematoxilina, principalmente para platelmintos. Los copépodos y nemátodos fueron transparentados con lactofenol. Los parásitos fueron identificados según la literatura citada en Muñoz \& Cortés (2009), además de literatura reciente (e.g., Castro \& Muñoz 2011, Muñoz \& Bott 2011).

Se calculó la similitud de las comunidades de parásitos entre las 4 especies de labrisómidos, mediante el índice de Jaccard, basado en la presencia y ausencia de especies parásitas, según su composición parasítica y mediante el índice de similitud de Bray-Curtis (McGarigal et al. 2000), considerando la abundancia promedio y la prevalencia de cada especie de parásito para cada especie de pez.

Se calculó el promedio de abundancia infrapoblacional y la prevalencia de cada especie parásita (Bush et al. 1997) y para la descripción de las comunidades se calculó el promedio de abundancia y riqueza infracomunitaria de parásitos (Bush et al. 1997). Adicionalmente, se calculó el promedio de la diversidad y uniformidad de Brillouin (Magurran 1988) de las infracomunidades de parásitos en cada especie de pez.

Para el análisis estadístico de los datos (tamaño de los hospederos y descriptores parasitológicos) se aplicó pruebas paramétricas. Para esto, los datos fueron previamente transformados a $\log _{10}(\mathrm{x})$ para obtener un mejor ajuste a la distribución normal y homogeneidad de varianza. Dado que la abundancia cero de parásitos afecta estos supuestos, sólo se consideró hospederos parasitados, de los cuales se obtuvo la intensidad, diversidad y uniformidad infracomunitaria de parásitos. Sin embargo, para la comparación de la riqueza de especies parásitas $\left[\log _{10}(x+1)\right]$ se consideró el total de hospederos, dado a que en este descriptor se lograron los supuestos indicados para pruebas paramétricas. Los descriptores parasitológicos, así como el tamaño de los hospederos (LT y PT), fueron comparados entre especies de peces a través de análisis de la varianza de una vía (ANDEVA) y fueron comparados en sus medias ajustadas con respecto a una covariable, longitud del hospedador, por medio del análisis de la covarianza (ANCOVA). Se realizaron correlaciones de Spearman para relacionar los descriptores infracomunitarios con la LT de los hospederos, para cada especie de pez y en conjunto.

\section{Resultados y Discusión}

De las 4 especies de peces se recolectó un total de 21 especies parásitas, 18 de las cuales estaban en $A$. microcirrhis, 12 en A. crinitus, 14 en A. variolosus y 4 en C. geniguttatus (Tabla 1). La sanguijuela no identificada Piscicolidae gen. sp. (morfoespecie 1, ver Moraga \& Muñoz 2010), el copépodo Holobomolochus chilensis y el digeneo Helicometrina nimia fueron las especies más abundantes y/o prevalentes (principalmente en Auchenionchus spp.) y las más comunes en las 4 especies de labrisómidos (Tabla 1). Ocho especies parásitas, correspondiente al $44 \%$ del total de especies encontradas, fueron compartidas entre las 3 especies de Auchenionchus.

La composición de las comunidades de parásitos en las especies Auchenionchus tuvo una similitud, basado en el índice de Jaccard, entre 42,8\% (A. microcirrhis vs A. crinitus) y 57,8\% (A. crinitus vs A. variolosus), mientras que Calliclinus geniguttatus tuvo una composición parasítica menos similar a la de los Auchenionchus spp., que osciló entre 22,2 y 28,1\%. Mientras que al considerar la composición y los datos numéricos de los parásitos, a través del índice de Bray-Curtis, se encontró que $A$. microcirrhis y A. crinitus fueron las especies más similares (69,1\% y 61,1\% de similitud parasítica en abundancia y prevalencia de las especies parásitas, respectivamente). Luego, la comunidad de parásitos de A. variolosus mostró un $61,2 \%$ de similitud en base a la abundancia, y un $32,7 \%$ de similitud considerando la prevalencia de las especies parásitas con respecto a $A$. microchrrhis y A. crinitus en conjunto. Finalmente, $C$. geniguttatus tuvo la similitud más baja, 38,5\% considerando la abundancia de parásitos y 15,2\% de similitud en la prevalencia de parásitos con respecto al grupo de los Auchenionchus (en conjunto).

El tamaño corporal de los peces obtenidos (LT y PT) fueron variables tanto dentro de una misma especie como entre las especies (Tabla 2). Auchenionchus microcirrhis 
Tabla 2. Promedio (X) con sus desviaciones estándares (DE) del tamaño corporal de peces (longitud total, LT y peso total, PT), y de los descriptores infracomunitarios de parásitos en 4 especies de labrisómidos, y comparación de estas 6 variables entre especies de peces a través de ANDEVA (F: estadígrafo, P: probabilidad estadística), y de 4 variables a través de ANCOVA, considerando a la LT como covariable / Average (X) and standard deviation (DE) of fish body size (total length, LT and weight, PT), and descriptors of the parasite infracommunities in 4 labrisomid species; and comparisons of these 6 variables among fish species through ANOVA (F: estadigraph, P: statistical probability), and ANCOVA using LT as covariable, for 4 variables

\begin{tabular}{|c|c|c|c|c|c|c|c|c|c|c|c|c|}
\hline \multirow{2}{*}{ Especies hospederas } & \multicolumn{2}{|c|}{ LT $(\mathrm{cm})$} & \multicolumn{2}{|c|}{ PT (g) } & \multicolumn{2}{|c|}{ Intensidad } & \multicolumn{2}{|c|}{ Riqueza } & \multicolumn{2}{|c|}{ Diversidad Brillouin } & \multicolumn{2}{|c|}{ Uniformidad } \\
\hline & $\mathrm{x}$ & $\mathrm{DE}$ & $\mathrm{x}$ & $\mathrm{DE}$ & $\mathrm{x}$ & $\mathrm{DE}$ & $\mathrm{X}$ & $\mathrm{DE}$ & $\mathrm{x}$ & $\mathrm{DE}$ & $\mathrm{x}$ & $\mathrm{DE}$ \\
\hline Auchenionchus microcirrhis & $8,6^{a, b}$ & 4,6 & $13,3^{\mathrm{a}}$ & 24,2 & $13,9^{a, b}$ & 23,8 & $1,9^{\mathrm{a}}$ & 1,8 & $0,31^{\mathrm{a}}$ & 0,37 & $0,40^{\mathrm{a}}$ & 0,42 \\
\hline Auchenionchus crinitus & $10,1^{a}$ & 3,6 & $16,5^{a, b}$ & 20,2 & $6,8^{a, b}$ & 6,7 & $2,0^{\mathrm{a}}$ & 1,3 & $0,34^{\mathrm{a}}$ & 0,31 & $0,55^{\mathrm{a}}$ & 0,44 \\
\hline Auchenionchus variolosus & $13,4^{\mathrm{c}}$ & 4,9 & $39,0^{a, b}$ & 33,7 & $32,5^{\mathrm{b}}$ & 58,2 & $4,1^{\mathrm{a}}$ & 3,5 & $0,61^{\mathrm{a}}$ & 0,51 & $0,57^{\mathrm{a}}$ & 0,42 \\
\hline Calliclinus geniguttatus & $5,0^{b}$ & 1,3 & $1,8^{\mathrm{c}}$ & 1,4 & $1,3^{\mathrm{c}}$ & 1,4 & $0,9^{\mathrm{b}}$ & 0,7 & $0,05^{\mathrm{b}}$ & 0,13 & $0,11^{b}$ & 0,29 \\
\hline \multicolumn{13}{|l|}{ Resultado ANDEVA } \\
\hline $\mathrm{F}_{(3,178)^{*}}$ & \multicolumn{2}{|c|}{6,44} & \multicolumn{2}{|c|}{6,66} & \multicolumn{2}{|c|}{3,17} & \multicolumn{2}{|c|}{2,75} & \multicolumn{2}{|c|}{3,53} & \multicolumn{2}{|c|}{4,80} \\
\hline$P$ & \multicolumn{2}{|c|}{$<0,001$} & \multicolumn{2}{|c|}{$<0,001$} & \multicolumn{2}{|c|}{0,026} & \multicolumn{2}{|c|}{0,044} & \multicolumn{2}{|c|}{0,017} & \multicolumn{2}{|c|}{0,003} \\
\hline \multicolumn{13}{|l|}{ Resultado ANCOVA } \\
\hline $\mathrm{F}_{(3,138)}$ & & & & & \multicolumn{2}{|c|}{2,06} & \multicolumn{2}{|c|}{0,39} & \multicolumn{2}{|c|}{0,54} & \multicolumn{2}{|c|}{0,42} \\
\hline$P$ & & & & & \multicolumn{2}{|c|}{0,108} & \multicolumn{2}{|c|}{0,753} & \multicolumn{2}{|c|}{0,654} & \multicolumn{2}{|c|}{0,740} \\
\hline
\end{tabular}

\footnotetext{
* Grados de libertad para la totalidad de los datos (LT, PT y riqueza de parásitos); $\mathrm{F}_{(3,138)}$ para la comparación de intensidad, diversidad y uniformidad de parásitos

Superíndices: letras distintas en los promedios de variables indican diferencias significativas entre los peces $(* P<0,05)$, basado en comparaciones a posteriori de Sheffeé
}

presentó mayor rango de LT (2,9-37,0 cm) debido al mayor tamaño muestral. Los ejemplares de Calliclinus geniguttatus fueron los más pequeños $(3,1-7,1 \mathrm{~cm})$, mientras que los ejemplares de $A$. variolosus fueron los más grandes (Tabla 2), con un rango de 4,8-19,9 cm. Consecuentemente, los promedios de LT y PT en A. variolosus fueron significativamente mayores que los de C. geniguttatus (Tabla 2).

La intensidad y riqueza parasítica aumentaron significativamente con la LT de Auchenionchus microcirrhis y A. crinitus (Fig. 1), pero no en A. variolosus (intensidad: rs $=0,09$ y riqueza: $\mathrm{rs}=0,38 ; P>0,05$ ) ni en $C$. geniguttatus (intensidad: $r s=-0,49$ y riqueza: $r s=-0,67 ; P$ $>0,05$ ). La diversidad (rs $=0,63 ; P<0,01$ ) y uniformidad (rs $=0,47 ; P<0,01$ ) de parásitos aumentaron significativamente con la LT sólo en A. microcirrhis, pero no en las otras 3 especies, en las cuales las correlaciones entre las variables mencionadas oscilaron entre $-0,40<$ rs $<0,36, P>0,05$. La abundancia, riqueza, diversidad y uniformidad de la infracomunidades de parásitos se correlacionaron positiva y significativamente con la LT de los peces individuales ( $\mathrm{n}=182$, rs $>0,45, P<0,01$ ), lo mismo se observó cuando se correlacionó el promedio de los descriptores parasitológicos con el promedio de la LT de cada una de las especies de peces $(n=4$, rs $>0,86, P<$ 0,001 , para los 4 descriptores parasitológicos). Por lo tanto, el tamaño corporal de los peces puede ser considerada con una variable influyente en los descriptores comunitarios de parásitos, ya que peces de mayor tamaño tuvieron más especies parásitas y con mayores abundancias.

El promedio de intensidad y riqueza infracomunitaria de parásitos fue distinta entre las especies de peces, siendo notoriamente mayor en Auchenionchus variolosus y menor en Calliclinus geniguttatus (Tabla 1), mientras que la riqueza, diversidad (índice de Brillouin) y uniformidad de parásitos fueron significativamente menor en C. geniguttatus en comparación con los otros peces (Tabla 2). Sin embargo, estas diferencias entre especies de peces fueron influidas por las distintas longitudes corporales que mostraron los peces analizados (Tabla 2), lo cual fue evidenciado en los resultados de ANCOVA, al introducir en el análisis comparativo la LT de los peces como covariable. De este modo, se encontró que las medias ajustadas de los 4 descriptores parasitológicos (abundancia, riqueza, diversidad y uniformidad) con respecto a la LT de los hospederos, no mostraba diferencias significativas entre especies de peces (Tabla 2), es decir, A. variolosus presentó mayor promedio de riqueza, abundancia y diversidad infracomunitaria de parásitos debido a su mayor tamaño corporal promedio en comparación con los otros peces, y a que los descriptores comunitarios de parásitos se relacionaron significativamente con el tamaño de los hospederos, lo que indica que, en general, las diferencias encontradas entre especies de peces sólo fue consecuencia de sus tamaños corporales. 
A

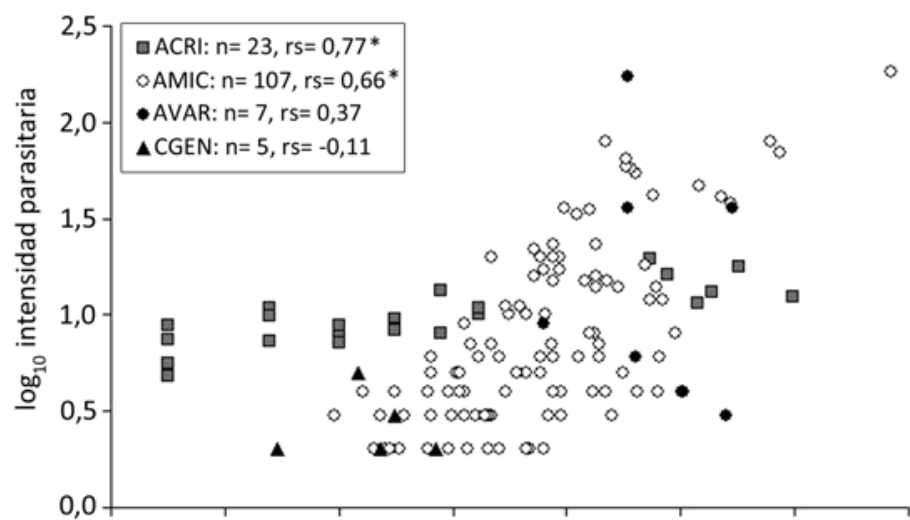

Figura 1. Correlación de Spearman entre los descriptores infracomunitarios de parásitos y la longitud total los hospederos, transformados a $\log _{10}$. A) Intensidad parasitaria y B) riqueza de especies parásitas. ACRI: A. crinitus, AMIC: A. microcirrhis, AVAR: $A$. variolosus, CGEN: C. geniguttatus, rs: Coeficiente de correlación, *: indica correlación significativa / Spearman correlation between infracommunity descriptors and the total length of each host species, transformed to $\log _{10}$. A) Parasitic intensity and $B$ ) species richness of parasites. ACRI: A. crinitus, AMIC: A. microcirrhis, AVAR: A. variolosus, CGEN: C. geniguttatus, rs: Correlation coefficient, *: indicates a significant correlation

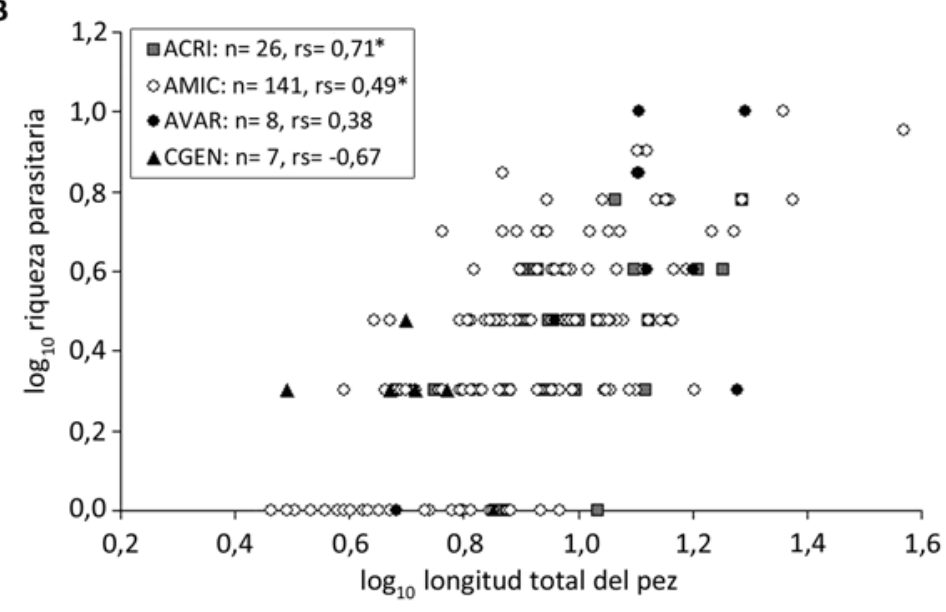

Los peces labrisómidos de Chile central presentaron una parasitofauna diversa (Tabla 1). Auchenionchus microcirrhis y A. variolosus fueron las especies con mayor riqueza componente de parásitos, 18 y 14 especies, respectivamente. Sin embargo, la mayoría de las especies parásitas de los labrisómidos eran generalistas, aunque todas ellas encontradas principalmente en peces intermareales. Por ejemplo, del total de especies parásitas registradas en los labrisómidos 13 han sido registradas en Scartichthys viridis (Muñoz \& Randhawa 2011), 8 en Helcogrammoides chilensis, 6 en Sicyases sanguineus, 5 en Girella laevifrons (Muñoz \& Delorme 2011) y 5 en Gobiesox marmoratus (Pardo-Gandarillas et al. 2004). Incluso varias de las especies de parásitos encontradas en los labrisómidos han sido más abundantes y prevalentes en otros peces intermareales (Muñoz \& Cortés 2009). Sin embargo, el digeneo adulto Prosorhynchoides carvajali (Bucephalidae) podría considerarse como la única especie parásita específica a los labrisómidos (índice de especificidad de 0,56 para abundancia y 0,60 para la prevalencia ${ }^{1}$ ), ya que ha sido registrada en A. microcirrhis y A. variolosus y ocasionalmente en Sicyases sanguineus siendo difícil que esta última especie adquiera el parásito con frecuencia debido a sus diferencias dietarias (Muñoz \& Bott 2011). Es decir, $P$. carvajali sería la única especie evolutivamente mejor adaptada a los labrisómidos.

Las especies congenéricas de peces labrisómidos (Auchenionchus spp.) tuvieron mayor similitud en composición parasitaria, tanto cualitativa (índice de Jaccard) como cuantitativamente (índice de Bray-Curtis), mientras que Calliclinus geniguttatus fue la especie menos similar entre los labrisómidos considerados. A pesar de que las diferencias en la composición de parásitos podría asociarse a los grados de parentesco entre las especies hospederas, i.e., especie congenéricas

${ }^{1}$ Indice de especificidad corregido propuesto por Rodhe \& Rodhe (2008). Datos no publicados de Muñoz \& Cortés, Universidad de Valparaíso, email contacto: gabriela.munoz@cienciasdelmar.cl 
más cercanas entre sí que la especie confamiliar, lo poco específico que resultaron los parásitos con respecto a sus hospederos labrisómidos, sugiere que las diferencias en las comunidades de parásitos de este grupo de peces sería consecuencia de factores ambientales. Por otro lado, la baja representación de parásitos en $C$. geniguttatus (Tabla 1) podría ser consecuencia de su pequeño tamaño corporal (Tabla 2) y tamaño muestral (Guégan \& Kennedy 1996).

En conclusión, las variaciones comunitarias de parásitos entre especies de hospederos observadas en este estudio, se deben principalmente a las diferencias del tamaño corporal de los peces, mientras que debido a la poca asociación de especie parásitas al linaje de los labrisómidos, las variaciones en composición parasitaria serían influidas por factores ambientales. Estos resultados, son similares a los de otros estudios, en cuanto a que factores ambientales y ecológicos de los hospederos tienen un rol importante en la estructura comunitaria de parásitos, y que a veces, parece ser más influyente que la relación filogenética entre los hospederos, como ha sido manifestado en estudios de temática similar en especies de peces Labridae (Muñoz et al. 2006), Paralichthyidae (Alarcos \& Timi 2012) y Gadiformes (Chávez et al. 2012).

\section{Agradecimientos}

Se agradece el financiamiento del proyecto FONDECYT 11060006. Los autores agradecen los comentarios y críticas de 3 evaluadores anónimos, que permitieron mejorar sustancialmente la versión original de este manuscrito.

\section{LITERATURA CITADA}

Alarcos AJ \& JT Timi. 2012. Parasite communities in three sympatric flounder species (Pleuronectiformes: Paralichthydae). Parasitology Research 110(6): 2155-2166.

Bush AO, KD Lafferty, JM Lotz \& AW Shostak. 1997. Parasitology meets ecology on its own terms: Margolis et al. revisited. The Journal of Parasitology 83(4): 575-583.

Castro R \& G Muñoz. 2011. Two new species of Colobomatus (Copepoda: Phylichthydae) parasitic on coastal fishes in Chilean waters. Crustaceana 84(4): 385-400.

Chávez RA, MT González, ME Oliva \& IM Valdivia. 2012. Endoparasite fauna of five Gadiformes fish species from the coast of Chile: host ecology versus phylogeny. Journal of Helminthology 86: 10-15.

Díaz P \& G Muñoz. 2010. Diet and parasites of the insular fish Scartichthys variolatus (Blenniidae) from Robinson
Crusoe Island, Chile: How different is this from two continental congeneric species? Revista de Biología Marina y Oceanografía 45(2): 293-301.

Flores K \& M George-Nascimento. 2009. Las infracomunidades de parásitos de dos especies de Scartichthys (Pisces: Blenniidae) en localidades cercanas del norte de Chile. Revista Chilena de Historia Natural 82: 63-71.

Guégan JF \& CR Kennedy. 1996. Parasite richness/ sampling effort/ host range: the fancy three-piece Jigsaw Puzzle. Parasitology Today 12: 367-369.

Iannacone J, JP Cerapio, J Cárdenas-Callirgos, K Sánchez, F Briceño \& A Dueñas. 2011. Comunidades de parásitos en el trambollo Labrisomus philippii (Steindachner, 1866) (Perciformes: Labrisomidae) de la zona costera de Chorrillos Lima, Perú. Neotropical Helminthology 5(1): 73-84.

Magurran AE. 1988. Ecological diversity and its measurements, 179 pp. Croom Helm, London.

McGarigal K, S Cushman \& S Stafford. 2000. Multivariate statistics for wildlife and ecology research, 279 pp. Springer-Verlag, New York.

McKenzie FE, GF Killen, JC Beier \& WH Bossert. 2011. Seasonality, parasite diversity, and local extinctions in Plasmodium falciparum malaria. Ecology 82(1): 2673-2681.

Moraga P \& G Muñoz. 2010. Prevalencia, abundancia y caracterización de morfoespecies de sanguijuelas (Annelida: Hirudinea) en peces intermareales de Chile central. Archivos de Medicina Veterinaria 42(2): 71-78.

Muñoz G \& N Bott. 2011. A new species of Prosorhynchoides (Trematoda, Bucephalidae) from the intertidal rocky zone of central Chile. Acta Parasitologica 56(2): 140-146.

Muñoz G \& Y Cortés. 2009. Parasite communities of a fish assemblage from the intertidal rocky zone of central Chile: Similarity and host specificity between temporal and resident fish. Parasitology 136: 1291-1303.

Muñoz G \& N Delorme. 2011. Variaciones temporales de las comunidades de parásitos de peces intermareales de Chile central: hospedadores residentes vs temporales. Revista de Biología Marina y Oceanografía 46(3): 313-327.

Muñoz G \& H Randhawa. 2011. Monthly variation in the parasite communities of the intertidal fish Scartichthys viridis (Blenniidae) from central Chile: are there seasonal patterns? Parasitology Research 109: 53-62.

Muñoz G \& L Zamora. 2011. Ontogenetic variation in parasite infracommunities of the clingfish Sicyases sanguineus (Pisces: Gobiesocidae). The Journal of Parasitology 91(1): 14-19.

Muñoz G, AS Grutter \& TH Cribb. 2006. Endoparasite communities of five fish species (Labridae: Cheilininae) from Lizard Island: How important is the ecology and phylogeny of the hosts? Parasitology 132: 363-373. 
Muñoz G, V Valdebenito \& M George-Nascimento. 2002. La dieta y la fauna de parásitos metazoos del torito Bovichthys chilensis Regan, 1914 (Pisces: Bovichthydae) en la costa de Chile centro-sur: variaciones geográficas y ontogenéticas. Revista Chilena de Historia Natural 75(4): 661-671.

Muñoz AA \& FP Ojeda. 1997. Feeding guild structure of a rocky intertidal fish assemblage in central Chile. Environmental Biology of Fishes 49: 471-479.

Muñoz AA \& FP Ojeda. 1998. Guild structure of carnivorous intertidal fishes of the Chilean coast: implications of ontogenetic dietary shifts. Oecologia 114: 563-573.

Oliva M \& JL Luque. 2002. Endohelminth parasites of the trambollo Labrisomus philippii (Steindachner) (Osteichthyes: Labrisomidae) from the central Peruvian coast. Comparative Parasitology 69(1): 100-104.

Pardo-Gandarillas MC, F Garcias \& M GeorgeNascimento. 2004. La dieta y la fauna de endoparásitos del pejesapo Gobiesox marmoratus Jenyns, 1842 (Pisces: Gobiesocidae) en el litoral de Chile están conectadas pero no correlacionadas. Revista Chilena de Historia Natural 77(4): 627-637.
Poulin R. 2007. Evolutionary ecology of parasites, 332 pp. Princeton University Press, Princeton.

Quijada P \& C Cáceres. 2000. Patrones de abundancia, composición trófica y distribución espacial del ensamble de peces intermareales de la zona centro-sur de Chile. Revista Chilena de Historia Natural 73(4): 739-747.

Rohde K \& P Rohde. 2008. How to measure ecological host specificity. Vie et Lilieu-Life and Environmental 58: 121-124.

Sapp KK \& GW Esch. 1994. The effects of spatial and temporal heterogeneity as structuring forces for parasite communities in Helisoma anceps and Physa gyrina. The American Midland Naturalist 132(1): 91-103.

Stephens J \& V Springer. 1973. Clinid fishes of Chile and Peru, with description of a new species, Myxodes ornatus, from Chile. Smithsonian Contributions to Zoology 159: 1-24.

Thieltges DW, KT Jensen \& R Poulin. 2008. The role of biotic factors in the transmission of free-living endohelminth stages. Parasitology 135(4): 407-426.

Zar JH. 1996. Biostatistical analysis, 718 pp. Prentice-Hall, Englewood Cliffs.

Editor Asociado: Marcelo Oliva M. 